PAPER • OPEN ACCESS

Two-dimensional correlation analysis for $\mathrm{x}$-ray photoelectron spectroscopy

To cite this article: S Li et al 2021 J. Phys. B: At. Mol. Opt. Phys. 54144005

View the article online for updates and enhancements.

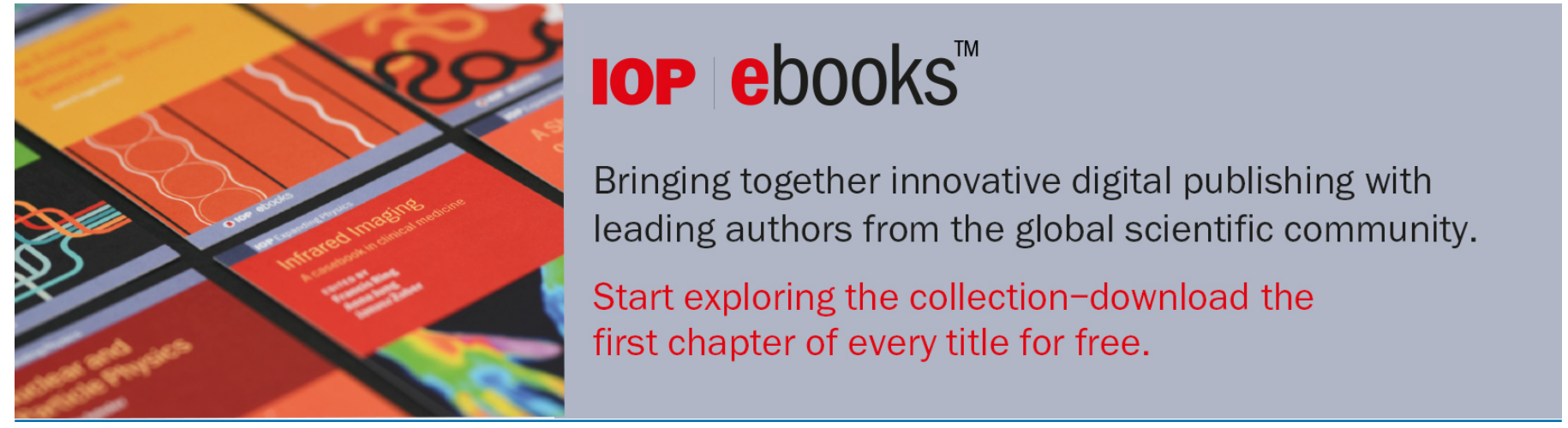

This content was downloaded from IP address 155.198 .10 .231 on $31 / 08 / 2021$ at 10:29 


\title{
Two-dimensional correlation analysis for $x$-ray photoelectron spectroscopy
}

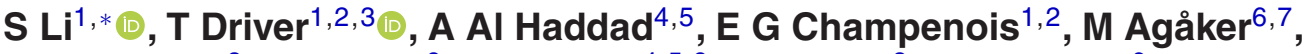 \\ O Alexander ${ }^{8}$, T Barillot $^{9}$, C Bostedt ${ }^{4,5,9}$, D Garratt ${ }^{8}$, L Kjellsson ${ }^{6}$ (D, \\ A A Lutman ${ }^{1}$, J-E Rubensson ${ }^{6}$, C Sathe ${ }^{7}$, A Marinelli $^{1}$, J P Marangos ${ }^{8}$ and \\ J P Cryan ${ }^{1,2,3}$ \\ 1 SLAC National Accelerator Laboratory, Menlo Park, CA 94205, United States of America \\ 2 Stanford PULSE Institute, Menlo Park, CA 94025, United States of America \\ ${ }^{3}$ Linac Coherent Light Source, SLAC National Accelerator Laboratory, 2575 Sand Hill Rd., Menlo Park, \\ California 94025, United States of America \\ ${ }^{4}$ Paul-Scherrer Institute, CH-5232 Villigen PSI, Switzerland \\ 5 Chemical Sciences and Engineering Division, Argonne National Laboratory, 9700 S. Cass Avenue, \\ Argonne, IL 60439, United States of America \\ 6 Department of Physics and Astronomy, Uppsala University, 75236 Uppsala, Sweden \\ 7 Max IV Laboratory, Fotongatan 2, 22484 Lund, Sweden \\ 8 The Blackett Laboratory Extreme Light Consortium, Imperial College London, London, SW7 2AZ, \\ United Kingdom \\ 9 LUXS Laboratory of Ultrafast Spectroscopy, Ecole Polytechnique Fédérale de Lausanne, Route \\ Cantonale, 1015 Lausanne, Switzerland \\ E-mail: siqili@slac.stanford.edu
}

Received 31 July 2020, revised 13 November 2020

Accepted for publication 25 November 2020

Published 12 August 2021

\begin{abstract}
$\mathrm{X}$-ray photoelectron spectroscopy (XPS) measures the binding energy of core-level electrons, which are well-localised to specific atomic sites in a molecular system, providing valuable information on the local chemical environment. The technique relies on measuring the photoelectron spectrum upon x-ray photoionisation, and the resolution is often limited by the bandwidth of the ionising $\mathrm{x}$-ray pulse. This is particularly problematic for time-resolved XPS, where the desired time resolution enforces a fundamental lower limit on the bandwidth of the $\mathrm{x}$-ray source. In this work, we report a novel correlation analysis which exploits the correlation between the x-ray and photoelectron spectra to improve the resolution of XPS measurements. We show that with this correlation-based spectral-domain ghost imaging method we can achieve sub-bandwidth resolution in XPS measurements. This analysis method enables XPS for sources with large bandwidth or spectral jitter, previously considered unfeasible for XPS measurements.
\end{abstract}

Keywords: x-ray photoelectron spectroscopy, free-electron laser, ghost imaging, AMO physics

(Some figures may appear in colour only in the online journal)

* Author to whom any correspondence should be addressed.

(c) (i) Original content from this work may be used under the terms of the Creative Commons Attribution 4.0 licence. Any further distribution of this work must maintain attribution to the author(s) and the title of the work, journal citation and DOI.

\section{Introduction}

The binding energy of core level electrons provides a sensitive measurement of the local electron density in a quantum system. These binding energies can be probed by analysing the electron kinetic energy spectrum produced by x-ray ionisation 
of the target. In this case, the binding energy (BE) is defined as

$$
\mathrm{BE}=\hbar \Omega-\mathrm{KE},
$$

where $\mathrm{KE}$ is the measured photoelectron kinetic energy and $\hbar \Omega$ is the $\mathrm{x}$-ray photon energy. In this type of measurement, the energy resolution is limited by the resolution of the electron kinetic energy measurement and the properties of the incident $\mathrm{x}$-ray pulse. The significant resolving power of high resolution electron spectrometers means that in standard $\mathrm{x}$-ray photoelectron spectroscopy (XPS) measurements, the bandwidth of the incident X-ray pulse is typically the primary limitation for energy resolution. This becomes problematic for time-resolved XPS measurements using ultrashort pulses, due to the large bandwidth required to support ultrashort (even sub-femtosecond) pulses. Moreover, this measurement scheme is challenging for $\mathrm{x}$-ray sources with inherent instabilities in shot-to-shot photon energy and requires accurate measurement and/or control of the central photon energy of the probing x-ray pulse.

These considerations pose a significant challenge for XPS measurements at X-ray free-electron laser (XFEL) sources. XFELs provide high-brightness, continuously tunable $\mathrm{x}$-ray pulses down to attosecond pulse durations [1], making them an ideal source for time-resolved measurements. The inherent bandwidth of few-to-sub femtosecond pulses strongly limits resolution regardless of source stability, and the inherent spectral jitter associated with self-amplified spontaneous emission (SASE) operation further degrades the available resolution for XPS measurements with XFELs.

In this paper, we present a correlation-based analysis method to perform XPS measurements with a fluctuating $\mathrm{x}$ ray source. By correlating single-shot measurements of the photoelectron kinetic energy spectrum and the incident x-ray spectrum, we are able to achieve sub-bandwidth resolution in the extracted XPS spectrum. Our technique exploits the shot-to-shot changes in incident $\mathrm{x}$-ray spectrum which are inherent to SASE operation of an XFEL. We demonstrate that the resolution of XPS measurement is in no way limited by the bandwidth of the incoming pulse, and is instead ultimately restricted by the measurable variation in the incoming X-ray spectrum. Although particularly amenable to XFEL measurements, the technique can in principle be applied to any photoelectron spectroscopy measurement using a noisy x-ray source.

The working principle of our method is based on 'ghost imaging'. In the spectral domain, the unknown absorption spectrum of a sample can be reconstructed by correlating two synchronous measurements: a reference measurement of the spectrum of the illuminating source, and a 'bucket' measurement of the total absorption through the sample. For onedimensional spectral ghost imaging, the bucket measurement consists of a single measurement. This total absorption can be measured from the depletion in the incident light pulse, or from a measurement of photo-products (electron, ion, or flourescence yield). Whilst this technique has been exploited in the spectral domain [2-7], it was originally developed for spatial imaging [8-19]. As the illuminating source fluctuates, so does the measured absorption. Thus, analysing the correlation between the coupled fluctuations of the incoming spectrum and the total sample absorption enables a reconstruction of the unknown absorption spectrum. Because ghost imaging exploits the fluctuations in the incident source, it has found many applications for XFELs, where shot-to-shot fluctuations are inevitable due to the stochastic nature of the SASE process. Extending beyond the spectral domain, it has also been proposed to use the ghost imaging technique in the time domain to conduct $\mathrm{x}$-ray pump/x-ray probe experiments within a single SASE pulse [20].

Returning to spectral domain ghost imaging measurements, we previously demonstrated sub-bandwidth $\mathrm{x}$-ray transient absorption spectroscopy using an attosecond XFEL pulse by applying the ghost imaging technique in the spectral domain [7]. In this example, the spectral reference measurement is derived from a measurement of the photoelectron kinetic energy spectrum far above an absorption edge, where the cross-section is nearly flat. We use the total yield of Auger-Meitner electrons as the bucket measurement. The correlation between these two synchronous measurements is then analysed to reconstruct the absorption spectrum. This measurement scheme paves the way for attosecond transient absorption experiments at XFEL facilities. The current work extends the one-dimensional technique to two dimensions, allowing us to recover the photon energy-resolved electron kinetic energy spectrum. What distinguishes the twodimensional analysis from any previous implementation, is the use of a photoelectron kinetic energy spectrum for each shot as a two-dimensional bucket measurement, instead of simply summing the total number of photoelectrons [7] or treating each x-ray emission energy bin independently [6].

\section{Experiment}

To demonstrate our method, we perform a single-shot measurement of the photoelectron kinetic energy spectrum from isopropanol in coincidence with the single-shot incident $\mathrm{X}$-ray spectrum at the Atomic Molecular Optical (AMO) experimental hutch at the Linac Coherent Light Source (LCLS) XFEL. The experimental layout is illustrated in figure 1. Soft X-ray photons centered at $\sim 502 \mathrm{eV}$ are generated by the XFEL and focused with a Kirkpatrick-Baez mirror pair into a dilute gaseous isopropanol sample, introduced via a $50 \mu \mathrm{m}$ diameter gas needle. Photoelectrons are collected with a hemispherical electron analyser (Scienta EW 4000). The microchannel plate (MCP) detector of the electron analyser is coupled to a phosphor screen and imaged with a single-shot CCD camera. After passing through the sample, the X-ray spectrum is analysed with an inline grating-based $\mathrm{x}$-ray spectrometer [21]. The spectrometer was provided by Uppsala University as an adapted version of the spectrometer described in reference [22], featuring a constant line spaced grating (1200 lines $\mathrm{mm}^{-1}$ ) in the Rowland geometry, which images a mechanical slit onto a MCP detector coupled to a phosphor screen. This phosphor screen is also imaged with a single-shot CCD camera.

The single-shot images from the hemispherical analyser are discriminated using a hit-finding algorithm and binned with 

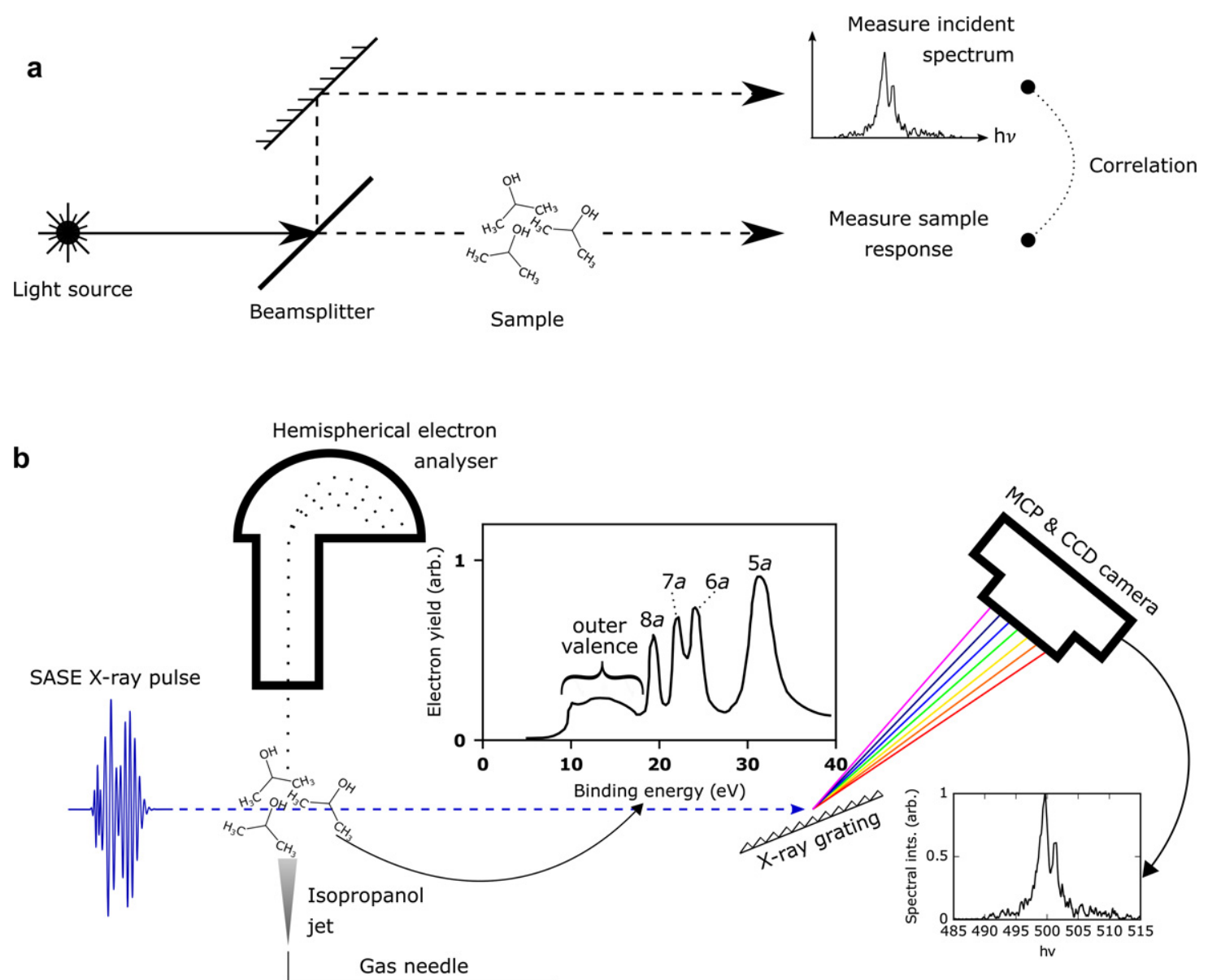

Figure 1. Panel (a) gives a schematic representation of ghost imaging experiments in the spectral domain. A noisy light source is split into two beams: reference (upper line) and signal, or bucket (lower line). The spectral content of the reference beam is analyzed and compared with a measurement of the signal beam. The signal beam is not spectrally dispersed, rather only the total transmission through the sample is recorded, hence the term, bucket. By comparing fluctuations in the transmission with changes in the incident spectrum, the absorption of the sample can be inferred. Panel (b) shows the experimental layout used in this work. SASE pulses at a central photon energy of $\sim 500 \mathrm{eV}$ are incident on gaseous isopropanol molecules introduced into the interaction chamber by an effusive gas needle. The photoelectrons are collected with a hemispherical electron analyser (Scienta EW4000). The incident x-ray spectrum is measured after the sample by a constant line spacing x-ray spectrometer. The low gas density and low interaction cross section for the isopropanol sample results in negligible absorption of the transmitted x-rays, thus the $\mathrm{x}$-ray spectrum at the photon spectrometer matches the incoming spectrum. The total absorption can be determined from the total electron yield measured in the electron analyzer.

$0.42 \mathrm{eV}$ resolution. The average detection rate was $\sim 36$ electrons/shot. Similarly the images from the x-ray spectrometer are discriminated, summed across the spatial dimension and binned with $0.44 \mathrm{eV}$ resolution. Examples of the processed single-shot photoelectron and $\mathrm{x}$-ray photon spectra are shown in the cascade plot in figure 2 . The bin size for both measurements is chosen to sufficiently resolve the features in the two-dimensional absorption spectrum, while keeping the computational time required for spectral reconstruction reasonable.

\section{Analysis}

This work is an extension of our previous implementation of spectral ghost imaging for x-ray absorption spectroscopy (XAS) with an attosecond XFEL pulse. The standard technique for absorption measurements at $\mathrm{X}$-ray facilities such as electron storage-rings and XFELs is to scan the incoming central photon energy across the region of interest and record the absorption at each wavelength by measuring the yield of photoproducts. The spectral resolution of these techniques is inherently limited by the bandwidth of the incident pulse. The recent demonstration of attosecond X-ray pulses at LCLS [1] opens the possibility for $\mathrm{X}$-ray pump/X-ray probe experiments at attosecond resolution using XAS and/or XPS. However, the bandwidth required to support pulses of attosecond duration (a 500 as FWHM light pulse has a minimum bandwith of $3.5 \mathrm{eV}$ FWHM) far exceeds the required resolution for a typical x-ray absorption or emission experiment $(\lesssim 1 \mathrm{eV})$. This limitation can be surpassed by measuring the incoming $\mathrm{x}$-ray spectrum in coincidence with the absorption (i.e. photoproduct yield) on a shot-to-shot basis, and then analyzing the correlations between the two measurements.

For low intensity $\mathrm{x}$-ray pulses, the $\mathrm{x}$-ray/matter interaction can be described accurately in a first-order perturbative expan- 


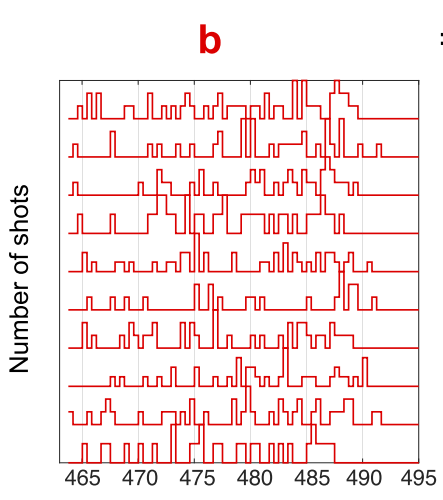

Electron kinetic energy $(\mathrm{eV})$
$=$

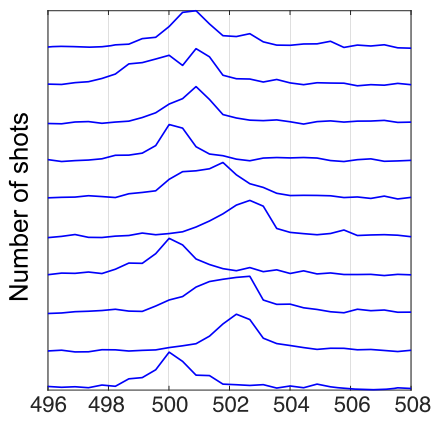

$h v(\mathrm{eV})$

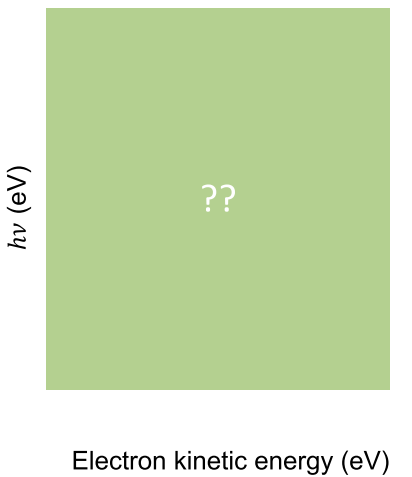

Figure 2. Schematic illustration of the two-dimensional spectral ghost imaging model. The single-shot electron spectra make up the rows of the two-dimensional bucket $\boldsymbol{b}$. Each spectrum is the product of the incoming photon spectra (which make up the rows of the matrix $\boldsymbol{A}$ ), and the two-dimensional target response function $\boldsymbol{x}$.

sion, where the absorption probability is given by the product of the cross-section and photon flux integrated over the $\mathrm{x}$ ray pulse. In this case, the yield of photoelectrons in a given kinetic energy bin is linearly proportional to the spectral intensity of the incoming x-ray pulse. For the $i$ th shot, the total yield of the photoelectrons $b_{i}$ can be written as a sum of the product between the $i$ th spectrum $A_{i}(\omega)$ and the unknown absorption spectrum, $x(\omega), b_{i}=\int \mathrm{d} \omega A_{i}(\omega) x(\omega) \sim \Sigma_{p} A_{i p} x_{p}$, where $\Sigma_{p}$ indicates discrete sum over the pixels in the x-ray spectrometer. When expanded to $n$ shots, we can modify this equation into a linear matrix multiplication:

$$
\boldsymbol{b}=\boldsymbol{A x},
$$

where $\boldsymbol{A}$ is an $n \times m$ matrix of the measured incident photon spectrum ( $m$-pixels) for each of the $n$ recorded shots, as shown in figure 1 . The vector $\boldsymbol{b}$ consists of the total integrated yield of photoproducts at each of the $n$ laser shots, and the unknown vector $\boldsymbol{x}$ describes the spectral response of the system. In the ghost imaging language used above, $\boldsymbol{A}$ is the reference measurement, and $\boldsymbol{b}$ is the bucket measurement. Solving equation (2) for $\boldsymbol{x}$ will then yield the absorption spectrum of the sample.

In this work we extend this technique to the case where $\boldsymbol{b}$ is an $n \times p$ matrix, by replacing the single pixel with a measurement consisting of $p$ electron kinetic energy bins. By discriminating the photoproducts according to electron kinetic energy, we reconstruct the XPS spectrum of the sample. For the case of a differential measurement of $\boldsymbol{b}$, the matrix equation, equation (2), is shown schematically in figure 2.

When cast as equation (2), the problem of achieving subbandwidth resolution in the $\mathrm{x}$-ray photoelectron spectrum by deconvolving the measured electron energy spectrum with the incident $\mathrm{x}$-ray spectrum becomes a matrix inversion problem. Ideally, we could write, $\boldsymbol{x}=\boldsymbol{A}^{-1} \boldsymbol{b}$, but in general, the matrix $\boldsymbol{A}$ is uninvertible. Despite this shortcoming, it is still possible to find a vector, $\boldsymbol{x}$, such that $\boldsymbol{x}$ minimises the function

$$
\|\boldsymbol{A x}-\boldsymbol{b}\|_{2}^{2}
$$

and thus provides the most probable value for $\boldsymbol{x}$ given the experimental measurement. In the absence of noise, this solu- tion can be rewritten as,

$$
\boldsymbol{x}=\left(\boldsymbol{A}^{T} \cdot \boldsymbol{A}\right)^{-1} \boldsymbol{A}^{T} \boldsymbol{b},
$$

where $\left(\boldsymbol{A}^{T} \cdot \boldsymbol{A}\right)^{-1} \boldsymbol{A}^{T}$ is called the Moore-Penrose pseudoinverse. While $\boldsymbol{A}^{-1}$ might not exist, $\left(\boldsymbol{A}^{T} \cdot \boldsymbol{A}\right)$ is a square matrix that typically has an inverse. However, equation (2) is an illconditioned problem, meaning that the solution $\boldsymbol{x}$ is acutely sensitive to noise in the measurements $\boldsymbol{A}$ and $\boldsymbol{b}$. The inevitable presence of experimental noise causes equation (4) to break down. This noise problem can be overcome through the process of regularisation: in addition to minimising the cost function (equation (3)) we can add additional constraints on the vector $\boldsymbol{x}$ in the minimisation process. For this work, we apply the knowledge that an XPS spectrum (or $\boldsymbol{x}$ ) is a sparse and positive definite matrix. In addition, we use the fact that for a physical system the second derivative of $\boldsymbol{x}$ along either the photon energy or electron kinetic energy is small, i.e. $\boldsymbol{x}$ is smooth.

We thus extend equation (3) to include regularisation as follows:

$$
\|\boldsymbol{A x}-\boldsymbol{b}\|_{2}^{2}+\lambda_{1}\|\boldsymbol{x}\|_{1}+\operatorname{Ind}_{+}(\boldsymbol{x})+\lambda_{2}\|\boldsymbol{L x}\|_{2}^{2},
$$

where the $\lambda$ 's are referred to as 'hyperparameters', whose values describe the relative importance of the different regularisation terms. The second term in equation (5) will minimise the $L_{1}$ norm (or the sum of the absolute values of the vector entries) to impose sparsity. In the third term, the Ind $_{+}$is the indicator function, which is 0 when the argument is non-negative and infinity otherwise. The final term in equation (5) will minimise the $L_{2}$ norm (or the sum of absolute value squared of the vector entries) of the second derivative of $\boldsymbol{x}$, which imposes smoothness. The second derivative operator is represented by the Laplacian matrix $\boldsymbol{L}$.

There are a number of existing algorithms to find the vector $\boldsymbol{x}$ that minimises equation (5). In this work, we employ an iterative algorithm, the 'alternating direction method of multipliers' (ADMM [23]), which independently solves for the individual terms in equation (5). In order to choose the optimal hyperparameters $\left(\lambda_{1}, \lambda_{2}\right)$ in equation (5) we simulate photoelectron spectra using an artificial 'ground truth' 

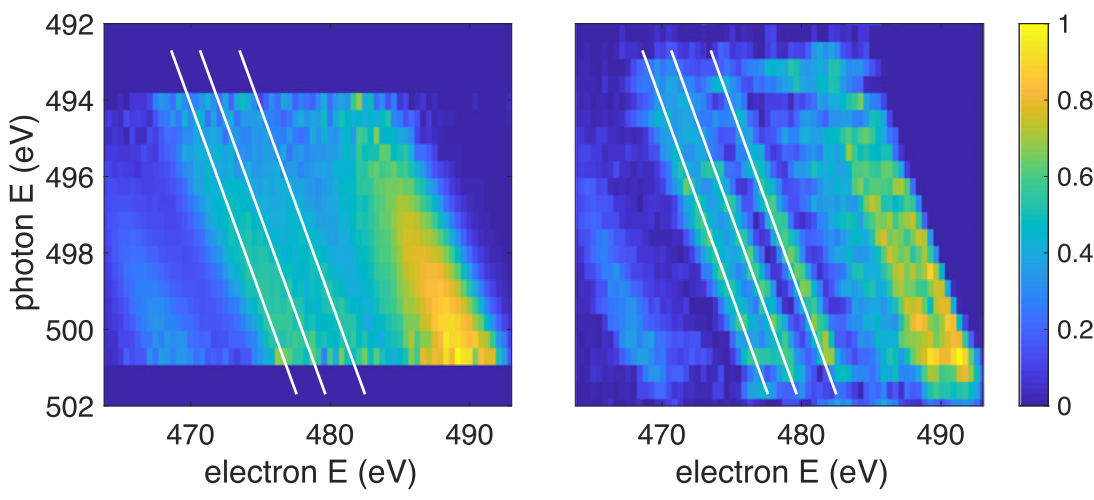

Figure 3. Two-dimensional reconstruction of the isopropanol spectral response, using the traditional method (left) and the spectral domain ghost imaging analysis (right). The white lines show the locations of $6 a, 7 a, 8 a$ triplet as measured by a monochromatized x-ray source in reference [24].

spectrum consisting of three Gaussian peaks. To generate the simulated photoelectron spectrum we multiply experimentally measured $\mathrm{x}$-ray spectra by this simulated ground truth spectrum according to equation (2). The resulting photoelectron spectrum is sampled according to a Poisson distribution with the same average electron count rate as measured in the experiment. The ADMM reconstruction is compared to the simulated ground truth for a wide range of hyperparameters. The hyperparameters giving the best agreement between the simulated and reconstructed spectra are then used in the reconstruction of the measured data. The success of the optimisation is weakly dependent on the hyperparameters, such that a change of an order of magnitude in any parameter does not significantly influence the results. We emphasise that our solution to equation (5), applies regularisation to the two-dimensional matrix, $\boldsymbol{x}$, as a whole, instead of treating each column individually as was done in reference [6]. In doing so, we are able to use all photoelectron and photon energy bins simultaneously to fully exploit the measured correlations across different shots.

The data analysed in this work consist of 128,132 laser shots, which were consecutively acquired in $\sim 15$ min. Using the same hyperparameters determined in the numerical simulations we run the ADMM algorithm on our dataset to produce the photoelectron spectrum shown in figure 3 . The dispersion of the photoelectron kinetic energy can be used to refine the photon energy calibration of the x-ray spectrometer. This is done by aligning the extracted photoelectron peaks with previous measurements made with a monochromatized $\mathrm{Al} K \alpha$ source [24].

\section{Discussion}

We can compare our reconstruction results with a traditional analysis scheme, which is obtained by sorting photoelectron kinetic spectra based on the central x-ray energy, as measured with the $\mathrm{x}$-ray spectrometer, and then averaging within each photon energy bin. The resolution of this measurement is limited by the inherent SASE bandwidth of the ionising Xray source, and the result is shown in the left hand panel of figure 3 . The spectral ghost imaging method (right hand panel of figure 3 ) is able to achieve sub-bandwidth resolution, clearly

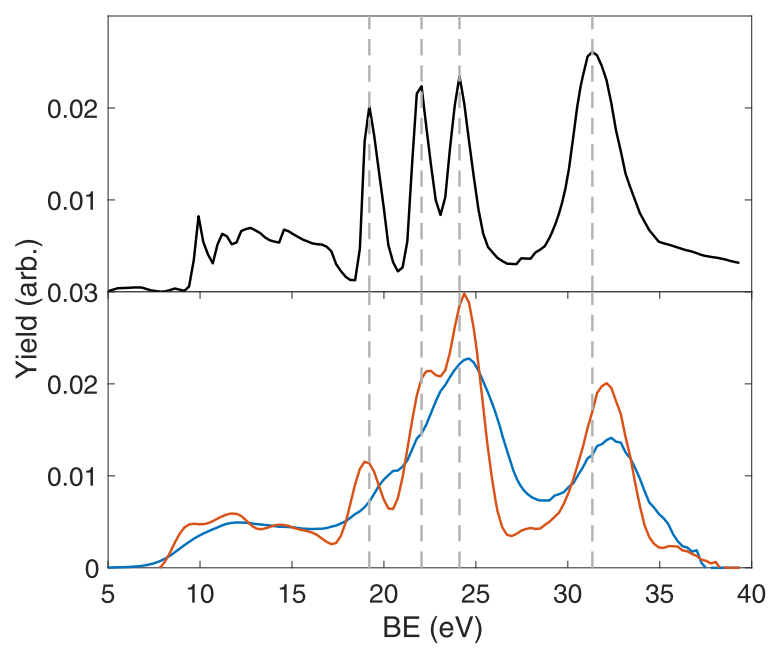

Figure 4. Retrieved electron binding energy for isopropanol. Top: previous measurement from reference [24] taken with a narrow bandwidth $\mathrm{x}$-ray source. Bottom: traditional XFEL XPS measurement (blue, see text) and spectral domain ghost imaging (red). We have corrected the transmission in the two bottom curves.

resolving the triplet structure $(475-485 \mathrm{eV}$ electron kinetic energy) associated with ionisation of the $6 a, 7 a$, and $8 a$ molecular orbitals, which are mostly carbon $2 s$ in character. In contrast, the traditional, SASE bandwidth-limited method recovers only a broad, unresolved feature in this region. In plotting our results in figure 3 we have corrected for the transmission of the electron spectrometer by dividing the reconstructed $x$ by an approximate kinetic energy-dependent transmission curve described in the appendix. We apply equation (1) to the solution $\boldsymbol{x}$ to plot the electron binding energy, summing across the photon energy axis to produce a one-dimensional trace, as shown in figure 4 . We compare our result with the measurement made by Nordfors et al using a monochromatised Al $K \alpha$ source [24] (top panel of figure 4). The energetic positions of the photoelectron peaks align well between the two measurements.

The result of the sorting method (red curve in figure 4) is a convolution between the true absorption spectrum and the $\mathrm{x}$ ray photon spectrum. When we apply the convolution theorem to deconvolve the one-dimensional result of the sorting method 


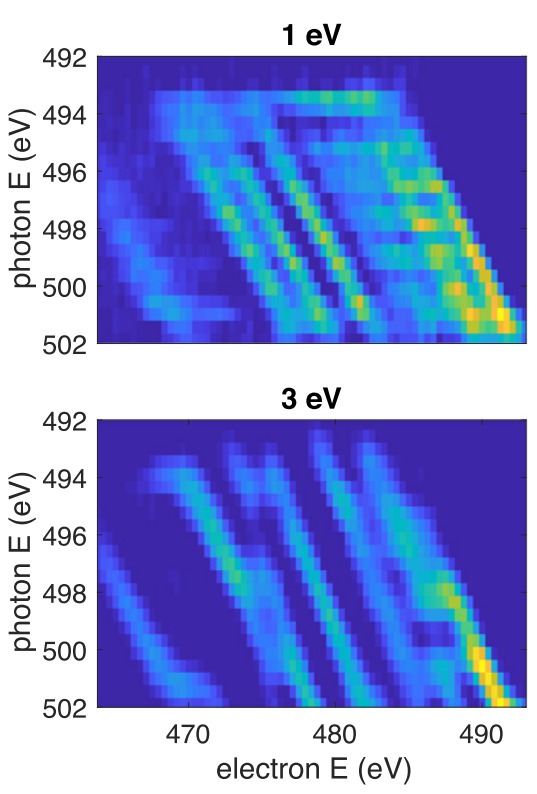

$2 \mathrm{eV}$

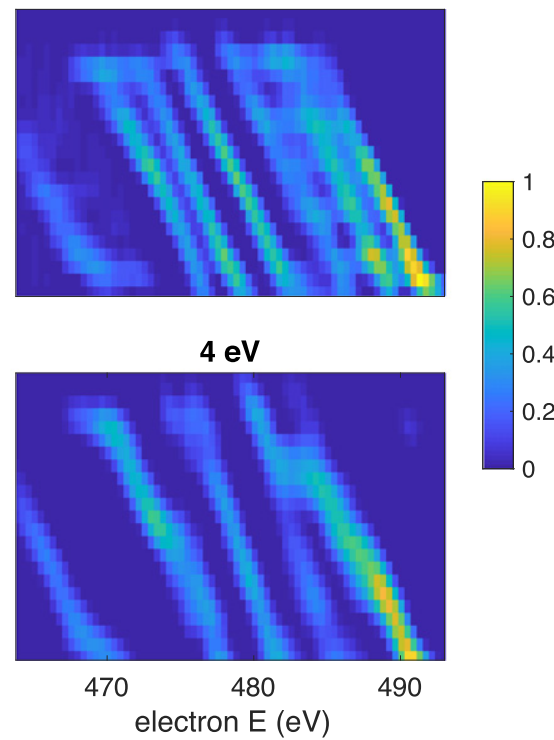

Figure 5. Effect of spectrometer resolution on spectral-domain ghost imaging reconstruction. The incident $\mathrm{x}$-ray spectrum is convolved with a Gaussian kernel with width of $1 \mathrm{eV}, 2 \mathrm{eV}, 3 \mathrm{eV}$, and $4 \mathrm{eV}$ respectively.
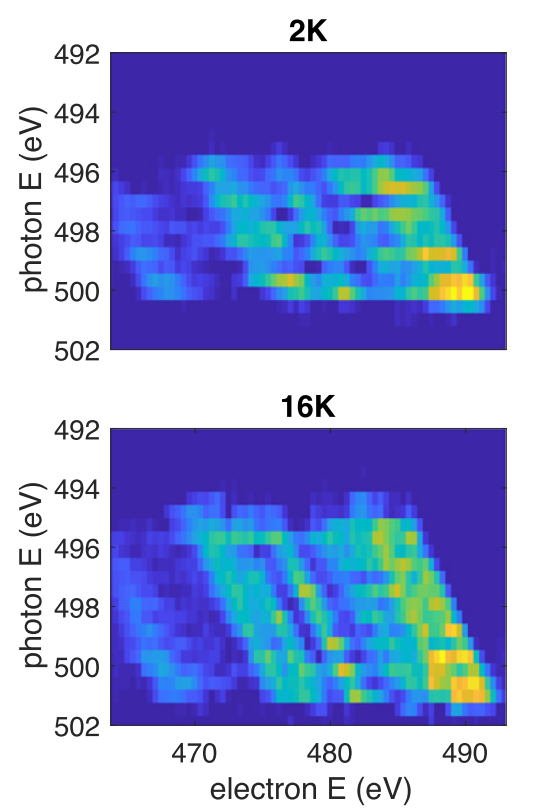

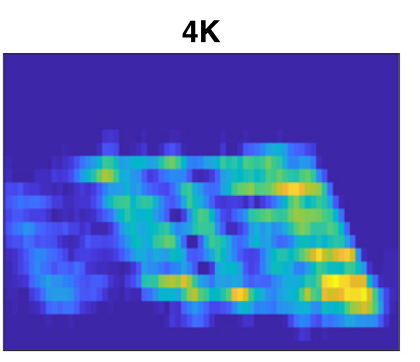

$32 \mathrm{~K}$

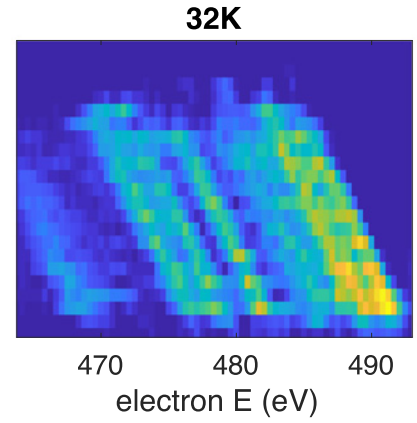

8K

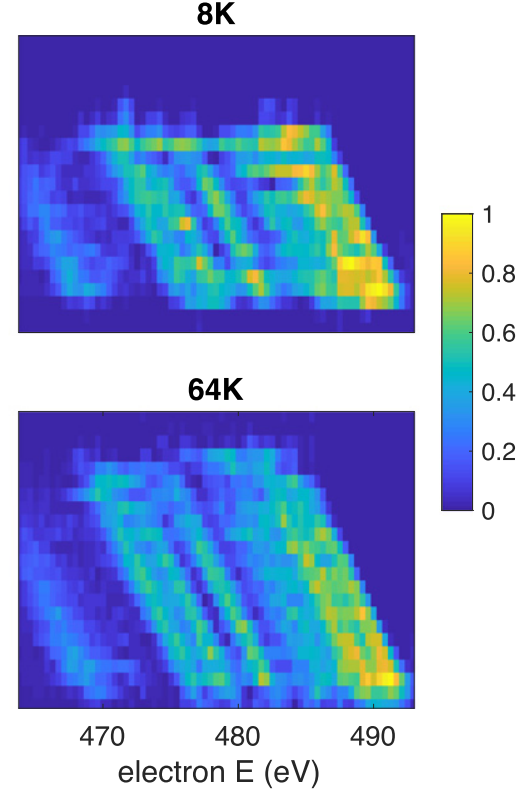

Figure 6. Effect of reduced statistics on the spectral-domain ghost imaging technique. A randomly selected subset of the data (number of shots denoted above each panel) is analysed according to equation (5).

it fails to capture the fine structures present in the absorption spectrum. This is because the deconvolution problem is also ill-conditioned and thus highly sensitive to noise. Our spectral ghost imaging method is, in essence, a deconvolution method particularly suited to XAS and XPS measurements by virtue of solving the noise problem through regularisation.

\section{Limitations of the correlation based approach}

The quantity of merit for the successful application of spectral ghost imaging is the amount of measurable variation in the shot-to-shot photon spectra. This quantity depends on the photon spectrometer resolution, the inherent fluctuations in the source, and the number of shots used in the reconstruction. The variation is most easily quantified by the 'correlation length', defined as the distance between pixels in the photon spectrometer which have a Pearson's correlation coefficient smaller than $1 / e$ [7]. As the correlation length decreases, fewer shots are needed for a quality reconstruction. An unintuitive corollary is that greater spectral resolution may be achieved in the XPS measurement by reducing the spectral stability of the ionising $\mathrm{x}$-ray source. Doing so allows the ionising source to more thoroughly sample the spectral response of a system within a given number of shots. In our measurements we do 

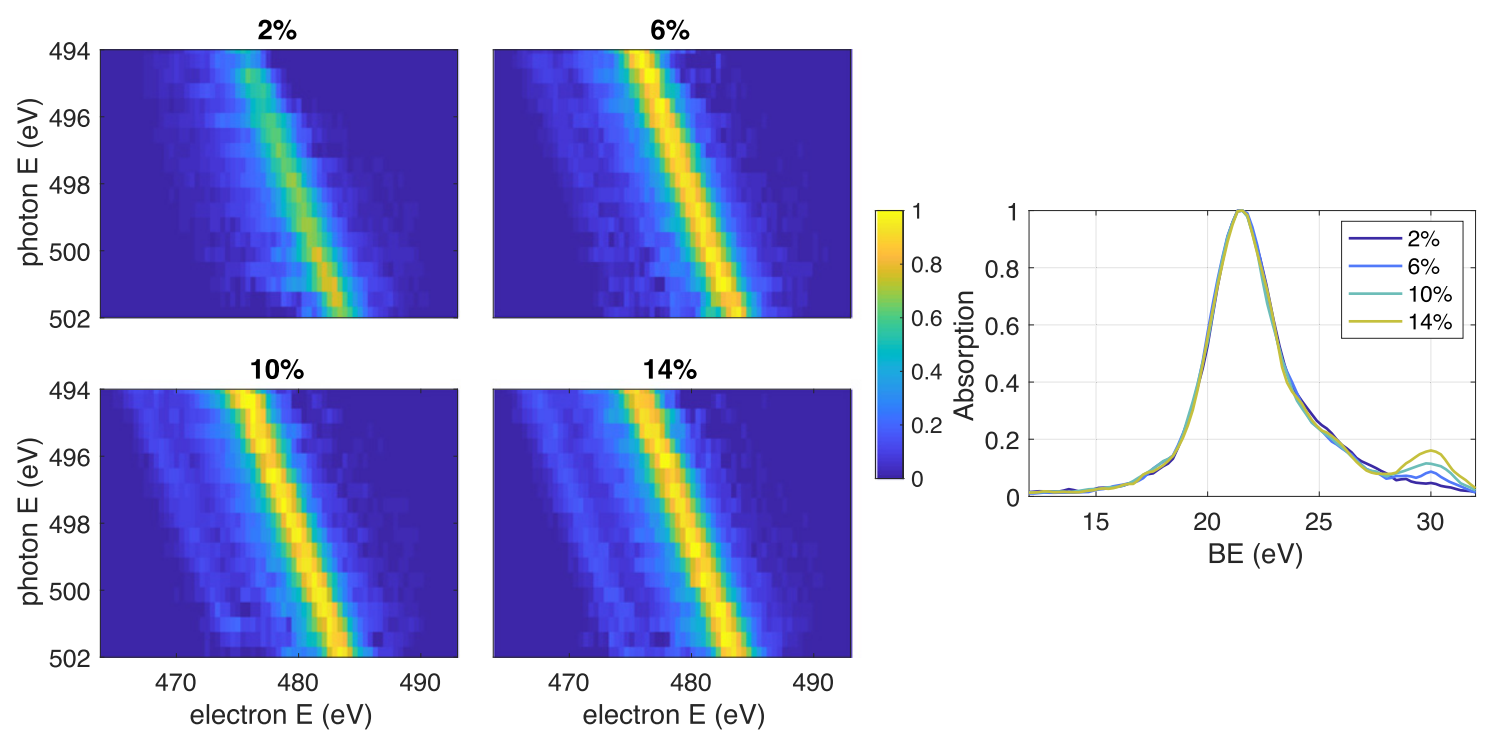

Figure 7. Applicability of spectral domain XPS to pump/probe studies. Spectral domain ghost imaging reconstruction with measured x-ray photon spectra and a simulated ground truth consisting of a primary Gaussian peak and a secondary Gaussian peak with a height at a small fraction of the main peak. We convert the $2 \mathrm{D}$ result to $1 \mathrm{D}$ binding energy plot and show the absorption against the binding energy on the right panel.

not intentionally introduce variation and instead rely on the natural fluctuations inherent to SASE operation. Initially the correlation length in the X-ray spectral measurement is $\sim 3.5$ pixels, corresponding to $\sim 1.6 \mathrm{eV}$. Next we study the independent effects of photon spectrum resolution and number of shots used on the resolution of the reconstructed measurement.

To study the effect of the spectrometer resolution, we convolve the measured photon spectra used to produce figure 3 with a Gaussian filter of increasing width $(\sigma)$ to simulate a degraded spectrometer resolution. The effect of decreasing spectral resolution is shown in figure 5, where increasing the Gaussian kernel width above $2 \mathrm{eV}$ leads to degradation in the resolution of the reconstruction. This level of degradation corresponds to a correlation length of $\sim 5$ pixels, and the effect worsens with increasing kernel width.

The effect of using fewer shots is also studied. The results shown in figure 3 are obtained by using 128, 132 individual shots. We randomly select, without replacement, a subset of the total number of measurements with size from 2000 to 64000 shots (figure 6). We note that the lower photon energy range $(<496 \mathrm{eV})$ starts to lose signal more quickly than the higher photon energy range, because the photon spectra have lower intensity in this region. When reducing the number of shots, any signal embedded in the low energy range suffers from insufficient measured variation as a result of the low flux in this range. Initially increasing the number of shots from 2000 to 4000 greatly improves the resolution in the reconstruction. This trend continues until $\sim 16000$ shots, above which level the resolution of the reconstruction is only slightly improved. The number of shots required for the reconstruction depends on the desired resolution and correlation length in the measured $x$-ray spectra, and so this number of $\sim 16000$ shots depends on the implementation.

It is worth noting, that there is another approach to enable higher resolution XPS measurements of gas phase molecules at FEL facilities, which involves employing an $\mathrm{x}$ ray monochromator to filter out a narrow bandwidth in wavelength [25]. The addition of the monochromator does not increase the spectral brightness of the source, but simply blocks all x-ray photons outside of a pre-defined bandwidth. The transmitted bandwidth needs to be much smaller than the bandwidth of the incoming spectrum in order to improve the spectral resolution. Therefore, in such a measurement, many photons in the natural SASE bandwidth are discarded (or wasted). In contrast, with spectral ghost imaging we are able to make use of all incoming $\mathrm{x}$-ray photons to interrogate the spectral response of our sample. With more incoming photons per shot, this method is able to produce a measurement at the same signal-to-noise ratio with fewer number of shots, which is tightly linked to the so-called Felgett's advantage [19].

\section{Application to time-domain measurements}

For spectral ghost imaging to be applied to time-resolved (pump/probe) XPS measurements, it is important to assess how the method resolves small signals on top of a large background. In a typical pump/probe experiment, only a small fraction $(1 \%-10 \%)$ of the sample is excited by the pump pulse (or 'pumped'). The XPS measurement then consists of this small, transient excited-state signal in addition to a large background due to ground-state or 'unpumped' molecules. It is the transient signal which carries the useful information on the time-dependent behaviour of the system. To study this situation, we conduct simulations where the simulated ground truth consists of one intense peak with a secondary neighboring peak of small relative intensity. As above, the photoelectron spectrum (bucket) is simulated by taking the matrix multiplication between the ground-truth and the measured photon 
spectra. To include the effect of shot noise in the photoelectron spectrum, we Poisson sample the calculated bucket with the same number of average photoelectrons as in the measured photoelectron spectra for the experiment described above. The results of this investigation are shown in figure 7 . We see that with a similar amount of noise as present in the measured photoelectron spectra and with the measured photon spectra, spectral ghost imaging is able to reconstruct the lower signal peak representing the excited-state signal. Resolving smaller fractional signals would require lower experimental noise in either the spectral measurement, the bucket measurement, or both.

\section{Conclusions}

In conclusion, in this work we have extended spectral ghost imaging at XFELs to a two-dimensional problem, where the spectral response of the sample is channel-resolved. In our particular implementation this allows for improved resolution in XPS measurements using an XFEL, where the inherent bandwidth and shot-to-shot variation in X-ray spectrum present challenges for high-resolution XPS measurements. The improved XPS measurements pave the way for the use of XPS as a time-resolved probe of localised electron density within a complex molecular system at XFEL facilities. Such measurements are a promising avenue for the observation of ultrafast phenomena such as charge migration and electron-nuclear coupling in molecules and condensed phase systems.

\section{Acknowledgments}

The authors would like to thank Thomas Wolf for useful discussion. This work was supported by US Department of Energy contract Nos. DE-AC02-76SF00515, DOE-BES Accelerator and detector research program Field Work Proposal 100317, DOE-BES, Chemical Sciences, Geosciences, and Biosciences Division, and Department of Energy, Laboratory Directed Research and Development program at SLAC National Accelerator Laboratory, under contract DE-AC02-76SF00515. CB and AA acknowledge support from the U.S. DOE-BES, Division of Chemical Sciences, Geosciences, and Biosciences through Argonne National Laboratory. Argonne is a U.S. Department of Energy laboratory managed by UChicago Argonne, LLC, under contract DE-AC02-06CH11357.

Use of the Linac Coherent Light Source (LCLS), SLAC National Accelerator Laboratory, is supported by the U.S. Department of Energy (DOE), Office of Science, Office of Basic Energy Sciences (BES) under Contract No. DE-AC02-76SF00515.

\section{Appendix. Transmission of the electron spectrometer}

The transmission function of the electron spectrometer plays an important role in determining the peak intensity in the

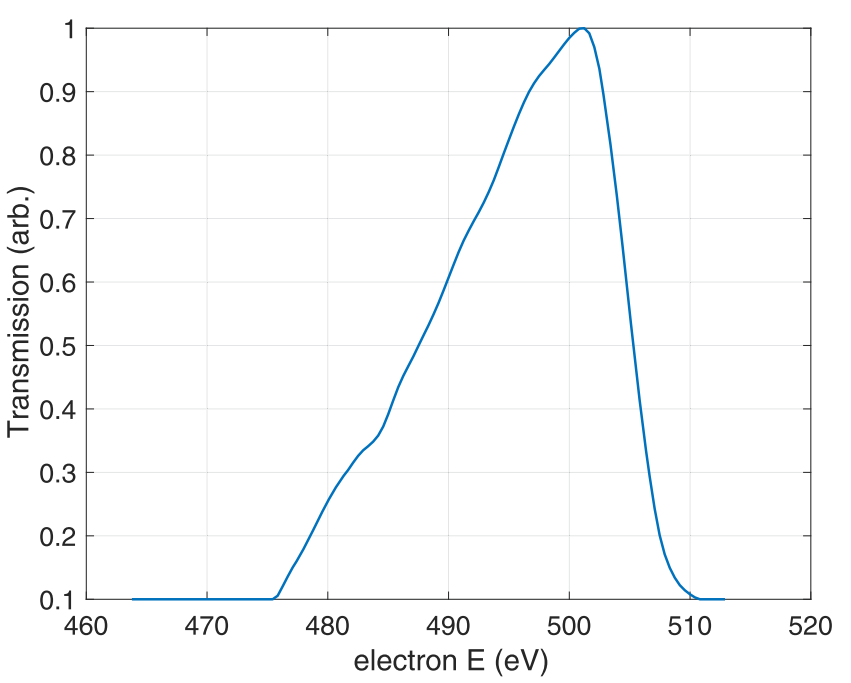

Figure A1. Transmission curve obtained from a separate spectral measurement of normal Auger-Meitner emission in the isopropanol molecule (see text for details).

reconstructed XPS spectrum. Unfortunately, we do not have a direct measurement of the transmission of the Scienta hemispherical electron analyser used in the measurement. Instead, we approximate the transmission function from a measurement of the normal (KVV) Auger-Meitner spectrum of isopropanol recorded with a photon energy of $\sim 600 \mathrm{eV}$. We convolve the Auger-Meitner electron kinetic energy spectrum with a broad $(10 \mathrm{eV})$ Gaussian to smooth away features in the Auger-Meitner spectrum, while retaining the coarse structure of the transmission curve. This reveals the characteristic triangular shape expected for these type of electron analyzers, as shown in figure A1. The Auger-Meitner spectrum is not reliable below $\sim 10 \%$, so we set a threshold at $10 \%$ and apply this transmission curve to the result of the spectral ghost imaging analysis. This estimate of the transmission is not perfect, but given the relative agreement between the peak intensities in our measurement along with the previous measurements of Nordfors et al, we believe it to be sufficient.

\section{ORCID iDs}

S Li (D) https://orcid.org/0000-0002-1262-6292

T Driver (D) https://orcid.org/0000-0002-3761-6883

L Kjellsson (D) https://orcid.org/0000-0001-9545-3579

J P Cryan (D) https://orcid.org/0000-0002-7776-0919

\section{References}

[1] Duris J et al 2020 Tunable isolated attosecond $\mathrm{x}$-ray pulses with gigawatt peak power from a free-electron laser Nat. Photon. 14 30-6

[2] Amiot C, Ryczkowski P, Friberg A T, Dudley J M and Genty G 2018 Supercontinuum spectral-domain ghost imaging Opt. Lett. 43 5025-8

[3] Janassek P, Blumenstein S and Elsäßer W 2018 Ghost spectroscopy with classical thermal light emitted by a superluminescent diode Phys. Rev. Appl. 9021001 
[4] Kalashnikov D A, Paterova A V, Kulik S P and Krivitsky L A 2016 Infrared spectroscopy with visible light Nat. Photon. 10 98

[5] Scarcelli G, Valencia A, Gompers S and Shih Y 2003 Remote spectral measurement using entangled photons Appl. Phys. Lett. 83 5560-2

[6] Kayser Y et al 2019 Core-level nonlinear spectroscopy triggered by stochastic X-ray pulses Nat. Commun. 10 1-10

[7] Driver T et al 2020 Attosecond transient absorption spooktroscopy: a ghost imaging approach to ultrafast absorption spectroscopy Phys. Chem. Chem. Phys. 22 2704-12

[8] Shapiro J H and Boyd R W 2012 The physics of ghost imaging Quantum Inf. Process. 11 949-93

[9] Erkmen B I and Shapiro J H 2010 Ghost imaging: from quantum to classical to computational Adv. Opt. Photon. 2405

[10] Duarte M, Davenport M, Takhar D, Laska J, Sun T, Kelly K and Baraniuk R 2008 Single-pixel imaging via compressive sampling IEEE Signal Proc. Mag. 25914730

[11] Pelliccia D, Rack A, Scheel M, Cantelli V and Paganin D M 2016 Experimental x-ray ghost imaging Phys. Rev. Lett. 117 113902

[12] Hong Y, Lu R, Han S, Xie H, Du G, Xiao T and Zhu D 2016 Fourier-transform ghost imaging with hard x rays Phys. Rev. Lett. 117113901

[13] Zhang A-X, He Y-H, Wu L-A, Chen L-M and Wang B-B 2018 Tabletop X-ray ghost imaging with ultra-low radiation Optica 5374

[14] Khakimov R I, Henson B M, Shin D K, Hodgman S S, Dall R G, Baldwin K G H and Truscott A G 2016 Ghost imaging with atoms Nature 540 100-3

[15] Schori A and Shwartz S 2017 X-ray ghost imaging with a laboratory source Opt. Express 25 14822-8
[16] Schori A, Borodin D, Tamasaku K and Shwartz S 2018 Ghost imaging with paired x-ray photons Phys. Rev. A 97 063804

[17] Li S, Cropp F, Kabra K, Lane T J, Wetzstein G, Musumeci P and Ratner D 2018 Electron ghost imaging Phys. Rev. Lett. 121114801

[18] Klein Y, Schori A, Dolbnya I P, Sawhney K and Shwartz S 2019 X-ray computational ghost imaging with single-pixel detector Opt. Express 27 3284-93

[19] Lane T J and Ratner D 2020 What are the advantages of ghost imaging? Multiplexing for x-ray and electron imaging Opt. Express 28 5898-918

[20] Ratner D, Cryan J P, Lane T J, Li S and Stupakov G 2019 Pumpprobe ghost imaging with SASE fels Phys. Rev. X 9011045

[21] Sanchez-Gonzalez A et al 2017 Accurate prediction of x-ray pulse properties from a free-electron laser using machine learning Nat. Commun. 8 1-9

[22] Nordgren J, Bray G, Cramm S, Nyholm R, Rubensson J E and Wassdahl N 1989 Soft x-ray emission spectroscopy using monochromatized synchrotron radiation (invited) Rev. Sci. Instrum. 60 1690-6

[23] Boyd S, Parikh N, Chu E, Peleato B and Eckstein J 2011 Distributed optimization and statistical learning via the alternating direction method of multipliers Found. Trends Mach. Learn. 3 1-122

[24] Nordfors D, Nilsson A, Mårtensson N, Svensson S, Gelius U and Ågren H 1991 X-ray excited photoelectron spectra of free molecules containing oxygen J. Electron Spectrosc. Relat. Phenom. 56 117-64

[25] Wolf T J A et al 2017 Probing ultrafast $\pi \pi * / n \pi *$ internal conversion in organic chromophores via $k$-edge resonant absorption Nat. Commun. 829 